\title{
EDITORIAL
}

\section{Does Board Certification Really Matter?}

\author{
Stuart T. Haines* \\ University of Maryland School of Pharmacy, Baltimore, Maryland
}

KeY Words board certification, pharmacy practice, outcomes.

(Pharmacotherapy 2014;34(8):799-802) doi: 10.1002/phar.1472

Quality gurus have long argued that if you get the structures and processes "right," good outcomes follow. ${ }^{1}$ A clinician's knowledge and skill is a fundamental structure of each health care encounter. Armed with a high level of technical expertise, a motivated and caring practitioner would be expected to implement a process of care that has the greatest likelihood of delivering the best possible outcomes. Board certification is intended to validate the practitioner's knowledge and skill, but some question its value or validity. ${ }^{2}$ Many factors influence outcomes. ${ }^{1}$ Proving that board certification makes a difference in terms of patient-oriented evidence that matters is no easy task.

Board certification is a voluntary process. It might be expected or desirable, but it is not mandated by law. Board certification is the norm among physicians and some specialty practice areas in nursing, like midwifery and anesthesia. ${ }^{3-5}$ For most physicians, board certification is valued due to economic incentives, enhanced employment opportunities, and social desirability. ${ }^{5}$ Ninety percent of physicians are board certified in the United States. ${ }^{3}$ In contrast, today, less than $10 \%$ of the pharmacy workforce in the United States is certified by the Board of Pharmacy Specialties (BPS) or the Commission on Certification in Geriatric Pharmacy. However, the number of board-certified pharmacists has steadily increased over the past 2 decades. Precisely what is fueling this growth is not entirely

*Address for correspondence: Stuart T. Haines, University of Maryland School of Pharmacy, 20 North Pine Street, Baltimore, MD 21201; e-mail: shaines@rx.umaryland.edu. (C) 2014 Pharmacotherapy Publications, Inc. understood, but many board-certified pharmacists indicate that becoming certified and maintaining the credential is important for their personal and professional development. 6,7 Some employers encourage board certification, but few make it a requirement of employment. ${ }^{7-9}$ In most settings, economic incentives for pharmacists to become board certified are not particularly strong, but some pharmacists report receiving onetime bonuses or reimbursement for board-certification test fees. ${ }^{6-8}$ A few states, notably North Carolina and New Mexico, as well as federal health agencies such as the Veterans Health Administration, officially recognize board certification as a qualifying credential for some advanced practice roles, but it is rarely mandated. ${ }^{6}$

Relatively little is known about employer perceptions of board-certified practitioners. In one study, most (86\%) nurse managers indicated that they would preferentially hire a certified nurse if all other qualifications were equal. ${ }^{10}$ Not surprisingly, managers who were themselves board certified were slightly more likely to favor hiring board-certified nurses than those nurse managers who were not board certified (91\% vs $83 \%$ ), but there was generally a strong preference regardless of the nurse manager's certification status or professional degree. Although most nurse managers (86\%) stated that certified nurses had a proven knowledge base, a commitment to lifelong learning $(76 \%)$, and performed better on the job (58\%), a minority (30\%) indicated that they assigned more complex patients to certified nurses.

Some pharmacy organizations strongly support board certification but not all. The American 
College of Clinical Pharmacy (ACCP) has been the most vocal in its support for board certification. ${ }^{11}$ Its policies make clear their belief that all pharmacists who are responsible for the management of patients with complex or special drug therapy needs should be board certified. ${ }^{12}$ Moreover, ACCP states that board certification should be required for all pharmacists who supervise students and residents during direct patient care experiences, teach students about therapeutics and drug therapy management, and deliver continuing education regarding drug therapy management. The American Society of HealthSystem Pharmacists (ASHP) has also supported board certification as an important quality metric. ${ }^{13}$ At the 2010 Pharmacy Practice Model Initiative summit, more than $80 \%$ of participants indicated their belief that board certification should be required for all pharmacists who provide drug therapy management to patients in specialty practice settings. ${ }^{14}$ The American Association of Colleges of Pharmacy encourages pharmacy practice faculty to become board certified. $^{15}$

Likewise, accrediting bodies in pharmacy also acknowledge the importance of board certification. The Accreditation Council for Pharmacy Education indicates that pharmacy practice faculty should possess credentials relevant to their practice and teaching responsibilities. ${ }^{16}$ ASHP's Commission on Credentialing requires postgraduate year 2 residency program directors to be board certified if certification in the area of specialization exists. $^{17}$

Although BPS is an autonomous division of the American Pharmacists Association (APhA), APhA does not have an explicit policy regarding board certification. ${ }^{18}$ APhA does have a number of policy statements regarding the pharmacist's responsibility to maintain continuing competence and engage in professional development activities. Maintaining board certification would certainly be consistent with those policies. Similar to APhA, the National Community Pharmacists Association and the National Association of Chain Drug Stores have no official policy regarding board certification for pharmacists.

Patients appear to value board certification, but misconceptions are common. A consumer survey commissioned by the American Board of Medical Specialties in 2010 found that 95\% of patients rated board certification important or very important, and 78\% would be bothered if their physician did not choose to maintain certification. ${ }^{19}$ Indeed, after bedside manner/commu- nication skills, patients indicated that board certification was the second most important factor in choosing a physician-more important than a family or friend's recommendation, location of the physician's practice, hospital affiliation(s), or school where the physician trained. Among women, board certification and bedside manner were equally valued, with 96\% rating both factors as important. Most respondents (78\%), however, incorrectly believed board certification is required to practice medicine, although most (66\%) understood that board certification is not the same as licensure. Only 23\% could correctly define the basic features of board certification. Nonetheless, many patients (39\%) would look for a new physician if they learned that their doctor was not board certified.

Although most patients and many practitioners, employers, and payers value board certification, a relative dearth of data are available regarding the real-world performance of boardcertified practitioners versus their noncertified peers. One of the earliest studies to examine the impact of hospital and physician characteristics on patient outcomes used data from the Hospital Cost and Utilization Project conducted at 373 participating nonfederal hospitals in $1977 .^{20}$ The investigators found that patients were more likely to survive an acute myocardial infarction if the attending physician was board certified and admitted patients to a teaching hospital. In 2002, a systematic review was published that examined whether a relationship exists between patient care outcomes and board certification; a positive association was found in approximately half of the included studies, but only $5 \%$ of the studies were sufficiently rigorous to answer the research question adequately. ${ }^{21}$ More recent studies have found that a physician's board certification status was associated with modest improvements in process of care measures following an acute myocardial infarction ${ }^{22}$ and that board certification scores were positively correlated with diabetes care process measures and patient satisfaction. ${ }^{23}$

In this issue of Pharmacotherapy, Dorsch et al provide much needed data regarding the potential benefits of board certification in pharmacy. ${ }^{24}$ Hospitals that employ pharmacists who are board-certified pharmacotherapy specialists and who have added qualifications in cardiology (AQCV) performed significantly better in terms of process of care metrics related to medication use than those hospitals that do not employ pharmacists with AQCV. Some might argue that 
hospitals that employ board-certified pharmacists are more likely to be big academic health science centers with the best available technology, better trained nursing staff, and a cadre of board-certified cardiologists all working together in well-defined specialty care units. Given these structural differences, we would expect large academic teaching hospitals to outperform small rural hospitals. The Dorsch et al study attempted to address these potential confounders by using a case-control methodology, matching hospitals based on geographic region and volume of patients discharged with cardiovascular diagnoses. $^{24}$ Although the authors also attempted to control for teaching hospital status, not surprisingly, hospitals that employed AQCV pharmacists were significantly more likely to be teaching hospitals. Nonetheless, a substantial proportion (more than 50\%) of the hospitals that did not employ AQCV pharmacists were teaching institutions. Thus it is unlikely that the results of the analysis can be explained by that factor alone. Other unseen, unaccounted, and perhaps unmeasurable qualities of the institutions that employed AQCV pharmacists might have influenced the results. For example, perhaps hospitals with AQCV pharmacists are generally more attuned to quality issues and have adopted a "quality culture." They may, therefore, be more likely to pay attention to quality metrics and implement improvements in a more timely manner. The fact that they employ a board-certified ACQV pharmacist is simply a byproduct of that culture. Note that we cannot assume institutions that did not employ AQCV pharmacists also did not employ board-certified pharmacists - indeed, these hospitals may have employed large numbers of board-certified pharmacists. All we know is that they did not employ any pharmacists with added qualifications in cardiology. Controlling for all potential confounding variables and conducting this type of research is exceedingly difficult.

To those who put considerable time and energy into earning and maintaining the credential, board certification is an important statement about their competence. The Dorsch et al study $^{24}$ affirms their belief that pharmacists who are board certified bring added value to an organization and should be given preferential treatment when it comes to employment and payment policy. Some employers and most patients agree-board certification is an important quality indicator. But few, it seems, are willing to pay extra for it. Skeptics contend that board certification is expensive and time consuming. ${ }^{3}$ Admittedly, it is. The evidence, they argue, is not sufficiently compelling to warrant such a costly investment. But we are unlikely to ever have evidence that conclusively proves that board-certified clinicians outperform noncertified ones. Yet we cannot ignore the expectations of patients and our health professional colleagues. Board certification will become increasingly important as public demand for greater accountability and quality intensifies. Does board certification really matter? I would say the answer is a qualified yes.

\section{References}

1. Donabedian A. The quality of care: how can it be assessed? JAMA 1988;260:1743-8.

2. Boukus E, Cassill A, O'Malley AS. A Snapshot of U.S. Physicians: key findings from the 2008 health tracking physician survey. Washington, DC: Center for Studying Health Change (HSC); 2009. Available from http://www.hschange.com/CONTENT/1078/1078.pdf. Accessed June 14, 2014.

3. Buscemi D, Wang H, Phy M, Nugent K. Maintenance of certification in internal medicine: participation rates and patient outcomes. J Community Hosp Intern Med Perspect 2012;2:19753. doi: http://dx.doi.org/10.3402/jchimp.v2i4.19753.

4. Fact Sheet: essential facts about midwives. Silver Spring, MD: American College of Nurse Midwives; 2014. Available from http://www.midwife.org/Essential-Facts-about-Midwives. Accessed June 14, 2014.

5. Certified Registered Nurse Anesthetists at a Glance. Available from http://www.aana.com/ceandeducation/becomeacrna/ Pages/Nurse-Anesthetists-at-a-Glance.aspx. Accessed June 14, 2014.

6. Pradel FG, Palumbo FB, Flowers L, Mullins CD, Haines ST, Roffman DS. White paper: value of specialty certification in pharmacy. J Am Pharm Assoc (2003) 2004;44:612-20.

7. Toussaint KA, Watson K, Marrs JC, Sturpe DA, Anderson SL, Haines ST. Prevalence of and factors that influence board certification among pharmacy practice faculty at United States colleges and schools of pharmacy. Pharmacotherapy 2013;33:105-11.

8. Romanelli F, Ryan M, Smith KM. Board of Pharmaceutical Specialties-certified faculty: a survey of United States colleges of pharmacy. Pharmacotherapy 2004;24:395-400.

9. Murphy JE, Hawkey L. Education, postgraduate training, board certification, and experience requirements in advertisements for clinical faculty positions. Am J Pharm Educ 2010;74:73.

10. Stromborg MF, Niebuhr B, Prevost S, et al. More than a title. Nurs Manage 2005;36:36-46.

11. Saseen JJ, Grady SE, Hansen LB, et al. Future clinical pharmacy practitioners should be board-certified specialists. Pharmacotherapy 2006;26:1816-25.

12. Haines ST, Andrus MR, Badowski M, et al. Board certification of pharmacist specialists. Pharmacotherapy 2011;31:11469.

13. American Society of Health-system Pharmacists. ASHP longrange vision for the pharmacy work force in hospitals and health systems: ensuring the best use of medicines in hospitals and health systems. Am J Health Syst Pharm 2007;64:1320-30.

14. American Society of Health-System Pharmacists. Executive Summary. Proceedings of the pharmacy practice model summit. Am J Health Syst Pharm 2011;68:e43-9.

15. Spinler SA, Bosso J, Hak L, et al. Report of the task force concerning board certification requirements for pharmacy practice faculty. Am J Pharm Educ 1999;61:213-6. 
16. Accreditation standards and guidelines for the professional program in pharmacy leading to the doctor of pharmacy degree. Standard 25, guidelines 25.1 Effective July 1, 2007.

17. ASHP Accreditation Standard for Postgraduate Year Two (PGY2) Pharmacy Residency Programs. Effective January 1, 2007.

18. American Pharmacists Association Policy Manual. Available from http://www.pharmacist.com/policy-manual. Accessed June 14,2014

19. News Release: 2010 ABMS Consumer Survey. Available from https://www.abms.org/News_and_Events/news_archive/ release_2010ABMSConsumerSurvey_04142011.aspx. Accessed June 14, 2014.

20. Kelly JV, Hellinger FJ. Heart disease and hospital deaths: an empirical study. Health Serv Res 1987;22:369-95.
21. Sharp LK, Bashook PG, Lipsky MS, Horowitz SD, Miller SH. Specialty board certification and clinical outcomes: the missing link. Acad Med 2002;77:534-42.

22. Chen J, Rathore SS, Wang Y, Radford MJ, Krumholz HM. Physician board certification and the care and outcomes of elderly patients with acute myocardial infarction. J Gen Intern Med 2006;21:238-44.

23. Hess BJ, Weng W, Holmboe ES, Lipner RS. The association between physicians' cognitive skills and quality of diabetes care. Acad Med 2012;87:157-63.

24. Dorsch MP, Lose JM, DiDomenico RJ. The effect of cardiovascular credentialed pharmacists on process measures and outcomes in myocardial infarction and heart failure. Pharmacotherapy 2014;34:803-808. 